\title{
Detection of CFIRB with AKARI/FIS Deep Observations
}

\author{
Woong-Seob Jeong ${ }^{a, *}$ Chris P. Pearson ${ }^{\text {a,b }}$ Hyung Mok Lee ${ }^{c}$ \\ Shuji Matsuura ${ }^{a}$ Mitsunobu Kawada ${ }^{d}$ Takao Nakagawa ${ }^{a}$ \\ Sang Hoon $\mathrm{Oh}^{\mathrm{c}}$ Mai Shirahata ${ }^{\mathrm{a}}$ Sungho Lee ${ }^{\mathrm{e}}$ \\ Ho Seong Hwang ${ }^{c}$ Hideo Matsuhara ${ }^{a}$ \\ ${ }^{a}$ Institute of Space and Astronautical Science, Japan Aerospace Exploration \\ Agency, Yoshinodai 3-1-1, Sagamihara, Kanagawa 229-8510, Japan \\ ${ }^{\mathrm{b}}$ ISO Data Centre, European Space Agency, Villafranca del Castillo, P.O. Box \\ 50727, 28080 Madrid, Spain \\ ${ }^{\mathrm{c}}$ Astronomy Program, Department of Physics and Astronomy, FPRD, Seoul \\ National University, Shillim-dong, Kwanak-gu, Seoul 151-742, South Korea \\ ${ }^{\mathrm{d}}$ Graduate School of Science, Nagoya University, Furo-cho, Chigusa-gu, Nagoya \\ 464-8602, Japan \\ ${ }^{\mathrm{e}}$ Korea Astronomy and Space Science Institute, 61-1 Whaam-dong, Yuseong-gu, \\ Daejeon 305-348, South Korea
}

\begin{abstract}
The Cosmic Far-Infrared Background (CFIRB) contains information about the number and distribution of contributing sources and thus gives us an important key to understand the evolution of galaxies. Using a confusion study to set a fundamental limit to the observations, we investigate the potential to explore the CFIRB with $A K A R I /$ FIS deep observations. The Far-Infrared Surveyor (FIS) is one of the focal-plane instruments on the $A K A R I$ (formerly known as ASTRO-F) satellite, which was launched in early 2006. Based upon source distribution models assuming three different cosmological evolutionary scenarios (no evolution, weak evolution, and strong evolution), an extensive model for diffuse emission from infrared cirrus, and instrumental noise estimates, we present a comprehensive analysis for the determination of the confusion levels for deep far-infrared observations. We use our derived sensitivities to suggest the best observational strategy for the $A K A R I / F I S$ mission to detect the CFIRB fluctuations. If the source distribution follows the evolutionary models, observations will be mostly limited by source confusion. We find that we will be able to detect the CFIRB fluctuations and that these will in turn provide information to discriminate between the evolutionary scenarios of galaxies in most low-to-medium cirrus regions.
\end{abstract}


Key words: Space-based infrared telescopes, Far infrared, Infrared cirrus, Evolution of extragalactic objects PACS: 95.55.Fw, 95.85.Gn, 98.58.Ca, 98.62.Ai

\section{Introduction}

Measurements of the infrared background have been carried out in several wavelength bands. The study of deep galaxy counts at infrared wavelengths has enabled us to resolve a significant fraction of the Cosmic Far-Infrared Background (CFIRB) which contains the accumulated intensity from redshifted and unresolved extragalactic sources. In addition to point sources, there also exist fluctuations in the surface brightness of extended structure due to neutral interstellar dust in the Milky Way that is heated by the interstellar radiation field, known as the infrared cirrus (Low et al., 1984). Moreover, although significant fluctuations from zodiacal emission have not yet been detected (Ábrahám, Leinert \& Lemke, 1997), this component may also contribute to the background fluctuations. Cirrus emission peaks at far-IR wavelengths but was detected in all 4 IRAS bands. The infrared emission from Galactic cirrus is usually a function of Galactic latitude and is serious for wavelengths longer than $60 \mu \mathrm{m}$. In many cases, the spatial power spectrum from fluctuations of the dust emission can be expressed as a simple power-law with the power index of -3 (Gautier et al., 1992). Unresolved sources below the detection limit also create fluctuations in the background, which was indeed detected from Infrared Space Observatory (ISO) observations (Lagache \& Puget, 2000; Matsuhara et al., 2000). By using the different properties of spatial fluctuations compared to unresolved sources and cirrus emission, some authors (Kiss et al., 2001; Miville-Deschênes, Lagache \& Puget, 2002; Grossan \& Smoot, 2006) have obtained significant levels of CFIRB fluctuation. However, since a dependency of the power index of the cirrus power spectrum on both the wavelength and surface brightness is suggested from recent observations (Kiss et al., 2003; Ingalls et al., 2004), we need to understand the spatial property of cirrus fluctuations as a function of Galactic latitude.

The Japanese AKARI (formerly known as ASTRO-F) satellite was launched on February 21st 2006 and is performing an all-sky survey (Nakagawa, 2001; Shibai, 2004; Pearson et al., 2004, 2007a) and deep pointed observations at the North and South Ecliptic Poles (Matsuhara et al., 2006) and other selected areas in 4 far-IR bands to much improved sensitivities, spatial resolutions and

\footnotetext{
* Corresponding author. Tel.: +81-42-759-8161; Fax: +81-42-786-7202.

Email address: jeongws@ir.isas.jaxa.jp (Woong-Seob Jeong).
} 
wider wavelength coverage than the previous IRAS survey over two decades ago. These far-IR observations will be expected to detect the CFIRB via fluctuation analysis. The actual observing plan may change depending on the in-flight performance of $A K A R I$. The purpose of this paper is to show the potential possible detection of the CFIRB and its expected fluctuation level.

\section{Source Counts Model and Confusion}

Source counts results in the far-IR regime from ISO and Spitzer observations (Matsuhara et al., 2000; Puget et al., 1999; Dole et al., 2004) show a significant evolution in the extragalactic population. The source count models used in this paper consider two evolutionary scenarios for galaxies revised from our previous luminosity evolution model (Pearson \& Rowan-Robinson, 1996) and burst evolution model (Pearson, 2001). For specific updated evolutionary parameters, see Pearson (2005, 2007d). In addition, a brief explanation and the color analysis for the revised models are described in Pearson et al. (2007b).

Due to the fluctuations from background structure and unresolved sources below the resolution of the telescope beam, the detection of sources is usually influenced by confusion effects. The sky confusion due to cirrus structures depends on sky position, however the source confusion due to unresolved sources remains constant everywhere except for clustering effects of sources and solar system objects. Since the cirrus fluctuations are represented by near-Gaussian noise, the final confusion limits $S_{\text {conf }}$ can be approximated by the summation of the two confusion noise components:

$$
S_{\mathrm{conf}}(\mathbf{r})=\sqrt{S_{\mathrm{cc}}(\mathbf{r})^{2}+S_{\mathrm{sc}}^{2}}
$$

where $S_{\mathrm{cc}}$ is the sky confusion limit due to cirrus structure, $S_{\mathrm{sc}}$ is the source confusion limit and $\mathbf{r}$ is the sky position. The source confusion estimated with the two evolutionary models of Pearson $(2005,2007 \mathrm{c})$ is $7.6-13 \mathrm{mJy}$ and $55-72$ mJy respectively (lower and upper limit is for luminosity evolution and burst evolution model, respectively), for the two wide bands of $A K A R I$ mission, i.e., WIDE-S and WIDE-L band with central wavelengths of $90 \mu \mathrm{m}$ and $140 \mu \mathrm{m}$. The sky confusion can be scaled with the cirrus surface brightness (Helou \& Beichman, 1990; Kiss et al., 2001; Kiss, Klaas \& Lemke, 2005; Jeong et al., 2005) and fig. 1 shows the final confusion limits for an assumed range of cirrus brightness levels for the $A K A R I$ mission. 


\section{Expected Cosmic Far-Infrared Background}

The flux levels from a randomly distributed population of extragalactic sources below the detection limit create fluctuations in the observed background. The CFIRB intensity $I_{\text {CFIRB }}$ and fluctuation $P_{\text {CFIRB }}$ produced by all sources with a flux below the limiting flux $S_{\text {lim }}$, are obtained from:

$$
I_{\mathrm{CFIRB}}=\int_{0}^{S_{\mathrm{lim}}} S \frac{d N}{d S} d S
$$

and

$$
P_{\text {CFIRB }}=\int_{0}^{S_{\lim }} S^{2} \frac{d N}{d S} d S,
$$

where $S$ is the flux and $d N / d S$ are the differential source counts. The detection limits $S_{\text {lim }}$ for $A K A R I$ estimated from our study including both sky confusion and source confusion (Jeong et al., 2005, 2006) with our revised source counts models (Pearson, 2005, 2007c) are shown in Figure 1. In the dark cirrus fields, the confusion limits are expected to be $7.7-13$ mJy for the WIDE-S band and $56-73 \mathrm{mJy}$ for the WIDE-L band (lower and upper values are for luminosity and burst evolution models, respectively).

Due to our better sensitivity, we can resolve more sources from the CFIRB compared to previous missions. Figs. 2 and 3 show the amount of the resolved CFIRB and the CFIRB fluctuation for the WIDE-S and WIDE-L bands. The amount of resolved CFIRB increases faster (i.e., decreasing of CFIRB fluctuation) in the WIDE-L band compared with that in the WIDE-S band. We list the expected CFIRB intensity, fluctuation and resolved fraction of CFIRB in Table 1. The expected $5 \sigma$ sensitivity for a diffuse source in AKARI's deep imaging mode (slow scan in $15 \mathrm{arcsec} / \mathrm{sec}, 2 \mathrm{sec}$ reset) is 0.34 and $0.56 \mathrm{MJy} / \mathrm{sr}$ for the WIDE-S and WIDE-L bands respectively (Verdugo et al., 2007). Though the CFIRB intensity expected in both evolutionary models is still below $3 \sigma$ sensitivity of the WIDE-S band, the CFIRB fluctuation in the burst evolution model is expected to be stronger than that of the noise $(\sim 600$ $\mathrm{Jy}^{2} / \mathrm{sr}$ at $\left.5 \sigma\right)$. However, the detector characteristics may also affect the accurate estimation of the fluctuation (Jeong et al., 2004; Shirahata et al., 2004). In the far-IR range, Lagache \& Puget (2000) and Matsuhara et al. (2000) have studied the detection of the CFIRB fluctuation from ISO observations in the Marano 1 field and the Lockman Hole, respectively. For comparison, we list our estimated fluctuations in Table 2. We find that the observed fluctuations are mostly located between the results predicted from our two evolutionary 
models except for the observations at $90 \mu \mathrm{m}$. Comparing with the model of Lagache, Dole \& Puget (2003), our estimated fluctuations from the burst evolution model are in good agreement. Since the deep observations of $A K A R I$ will have better sensitivity and resolution over a wider sky area than the ISO observations, we expect that we will be able to obtain more accurate fluctuation levels from our observations.

The estimation of fluctuations from the analysis of spatial power spectrum is usually used in the analysis of the CFIRB due to the lack of necessity for accurate absolute calibration. The power spectrum of cirrus emission at high Galactic latitudes (> 80 degree) also has a fluctuation of around $10^{6} \mathrm{Jy}^{2} / \mathrm{sr}$ at $0.01 \operatorname{arcmin}^{-1}$ and $160 \mu \mathrm{m}$ and a power index of $-2.9 \pm 0.5$. The shaded area in Figure 4 covers the fluctuations predicted by both evolutionary models. In the WIDE-L band, we may require a minimum areal coverage of $\sim 280-$ $400 \operatorname{arcmin}^{2}$ in order to effectively distinguish the CFIRB fluctuations in the observed data that will inevitably include both CFIRB and cirrus emission. If we use the WIDE-S band, the minimum required area coverage increases by a factor of $\sim 5$.

Obviously, as we observe higher cirrus regions, the detection limit becomes worse due to the increasing sky confusion noise, while the CFIRB fluctuation becomes larger (see the Figure 3). As can be seen in Figure 5, the fluctuations show a monotonic increase to medium cirrus regions. In the case of low Galactic latitude regions, since the cirrus fluctuation power should be more than $10^{8}$ (WIDE-S) and $10^{10}$ (WIDE-L) $\mathrm{Jy}^{2} / \mathrm{sr}$ at $0.01 \mathrm{arcmin}^{-1}$ (see Figure 4), it will be difficult to extract the CFIRB fluctuation from the analysis of the power spectrum (Note that this CFIRB fluctuation has a spatial scale close to the resolution of the detector). However, we expect to detect CFIRB fluctuations in most low-to-medium cirrus regions.

\section{Summary}

While the CFIRB fluctuation is expected to be almost constant irrespective of position on the sky, except for low Galactic latitude regions (due to severe sky confusion), cirrus fluctuations should be different depending on the regions of sky. We verified these results by comparing our simulated results with observational data from ISO (Kiss et al., 2001; Kiss, Klaas \& Lemke, 2005; Jeong et al., 2005). Thus, in order to estimate CFIRB fluctuations effectively, we need to compare the cirrus fluctuation via observations of regions at different Galactic latitudes (cirrus brightness). The deep observations planned with the AKARI mission will enable us to detect the CFIRB fluctuation in most regions of low-to-medium cirrus brightness in both the WIDE-S and WIDE-L bands. These observations will lead to a better understanding of the 
Cosmic Far-Infrared Background (CFIRB) - an important key to reveal the evolutionary scenario of galaxies in Universe.

\section{ACKNOWLEDGEMENTS}

Woong-Seob Jeong acknowledges a Japan Society for the Promotion of Science (JSPS) fellowship to Japan.

\section{References}

Ábrahám P., Leinert C., Lemke D. Search for brightness fluctuations in the zodiacal light at $25 \mu \mathrm{m}$ with ISO. Astron. Astrophys. 328, 702-705, 1997.

Dole, H., Lagache, G., Puget, J.-L. Predictions for cosmological infrared surveys from space with Multiband Imaging Photometer for SIRTF. Astrophys. J. 585, 617-629, 2003.

Dole, H., Le Floc'h, E., Pérez-González, P.G., et al. Far-infrared source counts at 70 and 160 microns in Spitzer deep surveys. Astrophys. J. Suppl. 154, 87-92, 2004.

Gautier, T. N. III, Boulanger, F., Pérault, M., Puget, J.L. A Calculation of confusion noise due to infrared cirrus. Astrophys. J. 103, 1313-1324, 1992.

Grossan, B., Smoot, C.F. Power spectrum analysis of Far-IR background in $160 \mu \mathrm{m}$ maps from the Multiband Imaging Photometer for Spitzer. Astron. Astrophys., submitted, astro-ph/0604512, 2006.

Helou G., Beichman C. A. The confusion limits to the sensitivity of submillimeter telescopes. Proc. of the 29th Liege International Astrophysical Coll., ESA Publ., 117-123, 1990.

Ingalls, J.G., Miville-Deschênes, M.-A., Reach, W.T., et al. Structure and Colors of Diffuse Emission in the Spitzer Galactic First Look Survey. Astrophys. J. Suppl. 154, 281-285, 2004.

Jeong, W.-S., Pak, S., Lee, H.M., et al. ASTRO-F/FIS observing simulation including detector characteristics. Adv. Space Res. 34, 573-577, 2004

Jeong, W.-S., Lee, H.M., Pak, S., et al. Far-infrared detection limits - I. Sky confusion due to Galactic cirrus. Mon. Not. R. Astron. Soc. 357, 535-547, 2005.

Jeong W.-S., Pearson C.P., Lee H.M., Pak S., Nakagawa T. Far-infrared detection limits - II. Probing confusion including source confusion. Mon. Not. R. Astron. Soc. 369, 281-294, 2006.

Kiss Cs., Ábrahám P., Klaas U., Juvela M., Lemke D. Sky confusion noise in the far-infrared: Cirrus, galaxies and the cosmic far-infrared background. Astron. Astrophys. 379, 1161-1169, 2001. 
Kiss, Cs., Ábrahám, P., Klaas, U., et al. Small-scale structure of the galactic cirrus emission. Astron. Astrophys. 399, 177-185, 2003.

Kiss, Cs., Klaas, U., Lemke, D. Determination of confusion noise for farinfrared measurements. Astron. Astrophys. 430, 343-353, 2005.

Lagache G., Puget J.L., 2000, A\&A, 355, 17

Lagache, G., Dole, H., Puget, J.L. Modelling infrared galaxy evolution using a phenomenological approach. Mon. Not. R. Astron. Soc. 338, 555-571, 2003.

Nakagawa T., 2001, in Pilbratt G.L., Cernicharo J., Heras A.M., Prusti T., Harris R., eds, ESA SP-460, The Promise of the Herschel Space Observatory, ESA Publications Division, p. 67

Low, F.J., Young, E., Beintema, D.A., et al. Infrared cirrus - New components of the extended infrared emission. Astrophys. J. Let. 278, L19-L22, 1984.

Matsuhara, H., Kawara, K., Taniguchi, Y., et al. ISO deep far-infrared survey in the "Lockman Hole". II. Power spectrum analysis: evidence of a strong evolution in number counts. Astron. Astrophys. 361, 407-414, 2000.

Matsuhara, H., Wada, T., Matsuura, S., et al. Deep Extragalactic Surveys around the Ecliptic Poles with AKARI (ASTRO-F). P. Astron. Soc. Jpn. 58, 673-694, 2006.

Miville-Deschênes, M.-A., Lagache, G., Puget, J.-L. Power spectrum of the cosmic infrared background at 60 and $100 \mu \mathrm{m}$ with IRAS. Astron. Astrophys. 393, 749-756, 2002.

Pearson, C.P., Rowan-Robinson, M. Starburst galaxy contributions to extragalactic source counts. Mon. Not. R. Astron. Soc. 283, 174-192, 1996.

Pearson, C.P. Evolutionary constraints from infrared source counts. Mon. Not. R. Astron. Soc. 325, 1511-1526, 2001.

Pearson, C.P., Shibai, H., Matsumoto, T., et al. ASTRO-F, super-IRAS, the All-Sky Infrared Survey. Mon. Not. R. Astron. Soc. 347, 1113-1129, 2004.

Pearson, C.P. Spitzer and ISO galaxy counts in the mid-infrared. Mon. Not. R. Astron. Soc. 358, 1417-1422, 2005.

Pearson, C.P., et al. A new light on cosmic evolution: The AKARI all-sky infrared survey. P. Astron. Soc. Jpn., submitted, 2007a.

Pearson, C.P., Jeong, W.-S., Matsuura, S., et al. Extragalactic population colour segregation in the AKARI deep SEP survey. Adv. Space Res. this issue, 2007b.

Pearson, C.P. A source count model for ISO, Spitzer, AKARI and beyond. Mon. Not. R. Astron. Soc. in preparation, 2007c.

Puget, J.L., Lagache, G., Clements, D.L., et al. FIRBACK. I. A deep survey at 175 microns with ISO, preliminary results. Astron. Astrophys. 345, 29-35, 1999.

Shibai, H. ASTRO-F mission. Adv. Space Res. 34, 589-593, 2004.

Shirahata, M., Matsuura, S., Makiuti, S., et al. Preflight performance measurements of a monolithic Ge:Ga array detector for the Far-Infrared Surveyor onboard ASTRO-F. in Mather J.C.(Ed.), Optical, Infrared, and Millimeter Space Telescopes Proc. SPIE, Bellingham, WA, Vol. 5487, 369-380, 2004. 
Verdugo, E., Yamamura, I., Pearson, C.P., et al. AKARI FIS data user manual ver.1.1 http://www.ir.isas.jaxa.jp/AKARI/Observation/, 2007. 
Table 1

Expected intensity, fluctuation and resolved fraction of CFIRB.

Luminosity evolution Burst evolution

WIDE-S WIDE-L WIDE-S WIDE-L

\begin{tabular}{ccccc}
\hline Intensity $(\mathrm{MJy} / \mathrm{sr})$ & 0.12 & 0.50 & 0.17 & 0.66 \\
Fluctuation $\left(\mathrm{Jy}^{2} / \mathrm{sr}\right)$ & 230 & 5300 & 660 & 9000 \\
Potential resolution $(\%)$ & 33 & 10 & 26 & 7 \\
\hline
\end{tabular}

Table 2

Comparison of CFIRB fluctuations.

\begin{tabular}{ccccccc}
\hline$\lambda$ & $\theta$ & $S_{\max }$ & Observations & Predicted & Predicted ${ }^{b}$ & Predicted (this work) $^{c}$ \\
$(\mu \mathrm{m})$ & $(\operatorname{arcmin})$ & $(\mathrm{mJy})$ & $\left(\mathrm{Jy}^{2} / \mathrm{sr}\right)$ & $\left(\mathrm{Jy}^{2} / \mathrm{sr}\right)$ & $\left(\mathrm{Jy}^{2} / \mathrm{sr}\right)$ & $\left(\mathrm{Jy}^{2} / \mathrm{sr}\right)$ \\
\hline 90 & $0.4-20$ & 150 & $13000 \pm 3000^{d}$ & 5300 & $2100-7200$ & $3200-4700$ \\
170 & $0.6-4$ & 100 & $7400^{e}$ & 12000 & $3800-14000$ & $8200-13000$ \\
170 & $0.6-20$ & 250 & $12000 \pm 2000^{d}$ & 16000 & $5500-18000$ & $11000-16000$ \\
\hline
\end{tabular}

${ }^{a}$ Model from Lagache, Dole \& Puget (2003).

${ }^{b}$ Lower and upper limits from our luminosity and burst evolution model, respectively (Jeong et al., 2006).

${ }^{c}$ Lower and upper limits from modified evolution models.

$d$ Matsuhara et al. (2000)

e Lagache \& Puget (2000) 

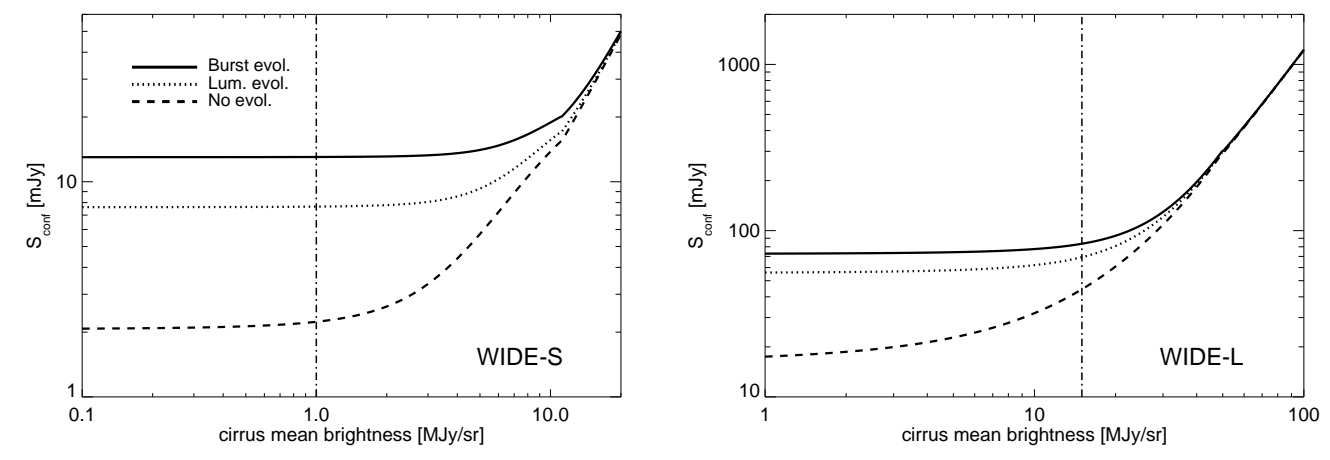

Fig. 1. Confusion limits considering both sky confusion and source confusion from careful simulations for the AKARI mission. The two vertical lines show the mean cirrus brightness $1.0 \mathrm{MJy} \mathrm{sr}^{-1}$ for the WIDE-S band (left) and $15 \mathrm{MJy} \mathrm{sr}^{-1}$ for the WIDE-L band (right), respectively. The leftwards and rightwards in the figure mean low and high cirrus regions, respectively. In low-level cirrus regions, source confusion is expected to dominate. Note that sky confusion increases as the mean cirrus brightness becomes larger while the source confusion has a constant value irrespective of the mean cirrus brightness.
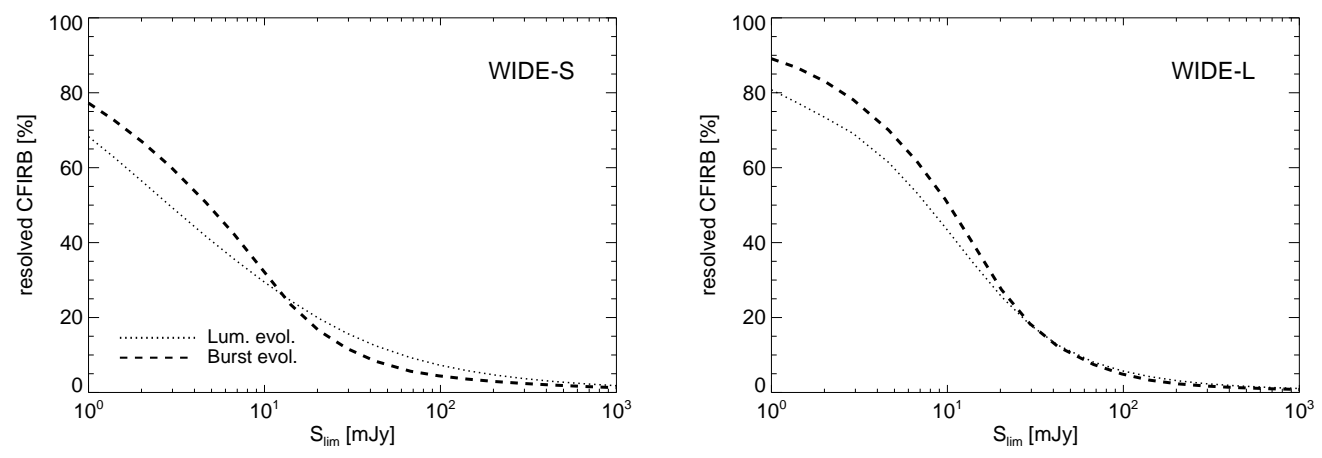

Fig. 2. Fraction of resolved CFIRB for the WIDE-S and WIDE-L bands. $S_{\lim }$ is the limiting sensitivity. 

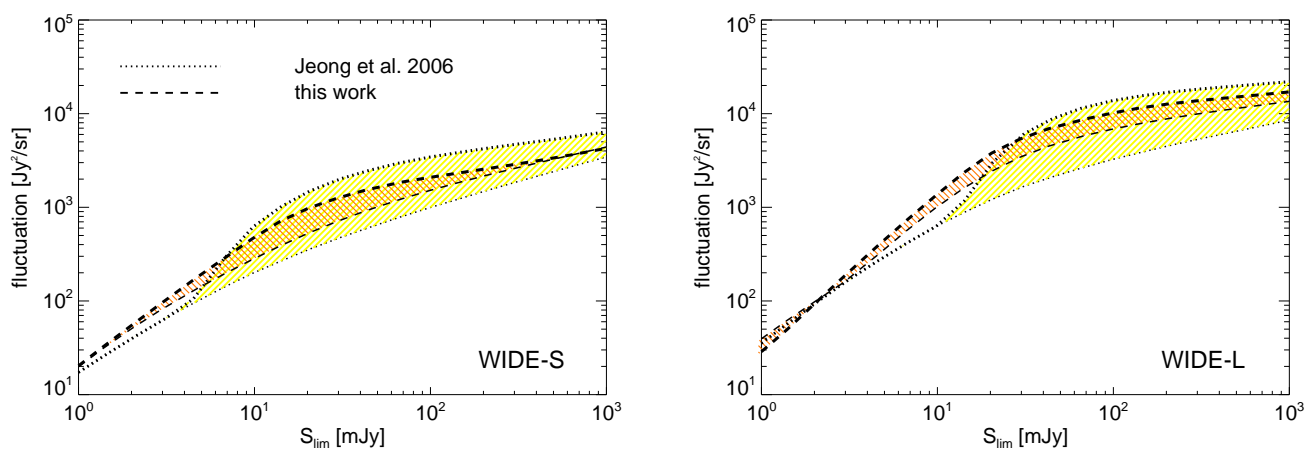

Fig. 3. Expected CFIRB and cirrus fluctuations in the WIDE-S and WIDE-L bands for the AKARI mission. The shaded area covers fluctuations from both evolutionary models. For comparison, we show the results for our revised and previous models. The lower limit is for the luminosity evolution model and the upper limit for burst evolution model.
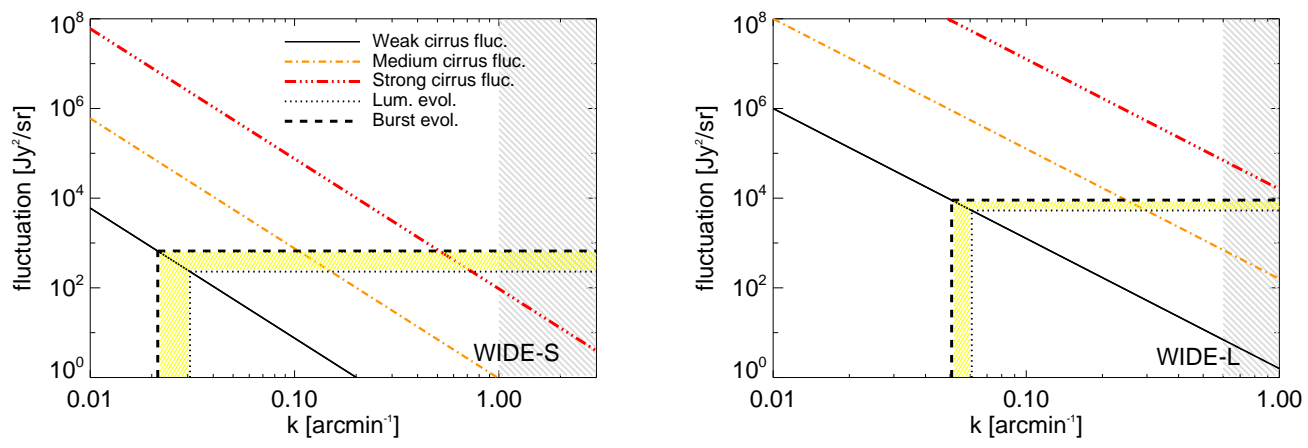

Fig. 4. Expected CFIRB and cirrus fluctuations in the WIDE-S and WIDE-L bands for $A K A R I$. The solid line shows the power spectrum of the cirrus emission at high Galactic latitude and the shaded area covers the fluctuations from the two evolution models. The lower limit is for the luminosity evolution model and the upper limit for the burst evolution model. The shaded area to the right means that those regions should be limited by the spatial resolution of the AKARI mission. 

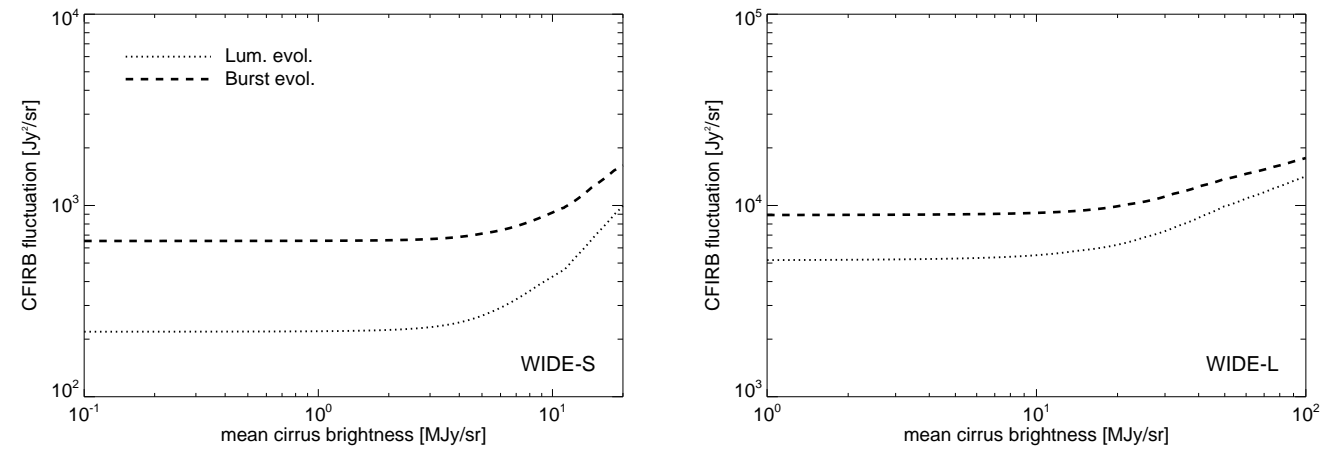

Fig. 5. Expected CFIRB fluctuation as a function of mean cirrus brightness in the WIDE-S and WIDE-L bands for the AKARI mission. The CFIRB fluctuation shows a monotonic increase in the medium mean cirrus brightness regions as the mean cirrus brightness increases. 